\title{
ASO Author Reflections: Is There Any Difference Among Various Gleason Scores Classified as Grade Group 4 Prostate Cancer?
}

\author{
Keiichiro Mori, $\mathrm{MD}^{1,2}$ (1) , Vidit Sharma, $\mathrm{MD}^{3,4}$, Shin Egawa, $\mathrm{MD}^{2}$, Derya Tilki, $\mathrm{MD}^{5,6}$, Stephen A. Boorjian, $\mathrm{MD}^{3}$, \\ and Shahrokh F. Shariat, MD ${ }^{1,7,8,9,10,11,12}$
}

${ }^{1}$ Department of Urology, Medical University of Vienna, Vienna, Austria; ${ }^{2}$ Department of Urology, The Jikei University School of Medicine, Tokyo, Japan; ${ }^{3}$ Department of Urology, Mayo Clinic, Rochester, MN; ${ }^{4}$ Department Of Urology, VA Health Services Research and Development Fellowship, University of California, Los Angeles, CA; ${ }^{5}$ Martini-Klinik Prostate Cancer Center, University Hospital Hamburg-Eppendorf, Hamburg, Germany; ${ }^{6}$ Department of Urology, University Hospital Hamburg-Eppendorf, Hamburg, Germany; ${ }^{7}$ Institute for Urology and Reproductive Health, Sechenov University, Moscow, Russia; ${ }^{8}$ Division of Urology, Department of Special Surgery, The University of Jordan, Amman, Jordan; ${ }^{9}$ Department of Urology, Weill Cornell Medical College, New York, NY; ${ }^{10}$ Department of Urology, University of Texas Southwestern, Dallas, TX; ${ }^{11}$ Karl Landsteiner Institute of Urology and Andrology, Vienna, Austria; ${ }^{12}$ Department of Urology, Second Faculty of Medicine, Charles University, Prague, Czech Republic

\section{PAST}

Currently, grade group (GG) 4 prostate cancer (PC) is equivalent to Gleason score (GS) 8 PC, which consists of GS $4+4,3+5$, and $5+3$. Grade group 4 still is considered a homogeneous entity with regard to its associated prognosis and treatment. However, some reports have raised questions as to whether GS 8 or GG 4 is a heterogeneous entity in terms of prognosis, and hence whether there is merit in reclassifying GG 4 into separate GGs. The authors have shown prognostic differences in patients who have $\mathrm{PC}$ in GG 4 treated with radical prostatectomy (RP) based on different Gleason scores for RP specimens, suggesting that considerable heterogeneity exists within GG 4 regarding oncologic and surgical pathologic outcomes. ${ }^{2}$ However, discrepancy exists between the biopsy and RP GS, with the two specimens matching exactly in approximately $50 \%$ of cases. ${ }^{3}$ Thus, it remains unclear whether our findings on RP specimens also hold true for biopsy specimens.

C The Author(s) 2021

First Received: 4 June 2021

Accepted: 9 June 2021;

Published Online: 21 June 2021

S. F. Shariat, MD

e-mail: shahrokh.shariat@meduniwien.ac.at

\section{PRESENT}

This study was conducted to investigate the prognostic differences between GS $3+5$, GS $4+4$, and GS $5+3$ in biopsy specimens from patients with PC classified as GG 4 based on the association with oncologic and surgical pathologic outcomes. ${ }^{4}$ In this multicenter retrospective study, 1791 patients (GS 3+5: 190; GS 4+4: 1557; and GS 5+3: 44) with biopsy GG 4 were included. During a median follow-up period of 75 months, 750 patients experienced biochemical recurrence (BCR), 146 died of any cause, and 57 died of PC. The results indicate that GS $5+3$ was associated with significantly higher rates of GS upgrading in RP specimens than GS $3+5$ or GS $4+4$. In contrast, no association was found between GS and lymph node metastases, non-organ-confined disease, positive surgical margin, or extraprostatic extension disease. Moreover, GS was not associated with overall survival or cancer-specific survival, but was associated with BCR-free survival $(P=0.03)$.

\section{FUTURE}

In this study, the patients with biopsy GG 4 exhibited some limited but clinically significant heterogeneity. Indeed, significant differences were seen in association with GS upgrading, downgrading, and BCR. The absence of central reviews involving expert pathologists is 
a limitation of this study given that earlier studies lacking central reviews indeed were associated with high proportions of patients with GS $3+5$ and GS $5+3$ and that a high percentage of GS $3+5$ and GS $5+3$ has been re-categorized upon expert review. Well-designed prospective studies with prolonged follow-up evaluation and central pathologic review are warranted to validate the differential prognostic and biologic impact of the different GS within GG 4 in the clinical setting.

FUNDING Open access funding provided by Medical University of Vienna.

DISCLOSURE There are no conflicts of interest.

OPEN ACCESS This article is licensed under a Creative Commons Attribution 4.0 International License, which permits use, sharing, adaptation, distribution and reproduction in any medium or format, as long as you give appropriate credit to the original author(s) and the source, provide a link to the Creative Commons licence, and indicate if changes were made. The images or other third party material in this article are included in the article's Creative Commons licence, unless indicated otherwise in a credit line to the material. If material is not included in the article's Creative Commons licence and your intended use is not permitted by statutory regulation or exceeds the permitted use, you will need to obtain permission directly from the copyright holder. To view a copy of this licence, visit http://creativecommons. org/licenses/by/4.0/.

\section{REFERENCES}

1. Mori K, Miura N, Comperat E, et al. A systematic review and meta-analysis of prognostic impact of different Gleason patterns in ISUP grade group 4. Minerva Urol Nephrol. 2021;73(1):42-49.

2. Mori K, Sharma V, Comperat EM, et al. Differential prognostic impact of different Gleason patterns in grade group 4 in radical prostatectomy specimens. Eur J Surg Oncol. 2021;47(5):1172-1178

3. 3. Kvåle R, Møller B, Wahlqvist R, et al. Concordance between Gleason scores of needle biopsies and radical prostatectomy specimens: a population-based study. BJU Int. 2009;103:1647-54.

4. Mori K, Sharma V, Comperat EM, Sato S, Laukhtina E, Schuettfort VM, et al. Prognostic impact of different Gleason patterns on biopsy within grade group 4 prostate cancer. Ann Surg Oncol. 2021. https://doi.org/10.1245/s10434-021-10257-x.

5. 5. Kryvenko ON, Williamson SR, Schwartz LE, Epstein JI. Gleason score $5+3=8$ (grade group 4) prostate cancer: a rare occurrence with contemporary grading. Hum Pathol. 2020;97:40-51.

Publisher's Note Springer Nature remains neutral with regard to jurisdictional claims in published maps and institutional affiliations. 